\title{
Inter-platform Applications
}

Communications between Control Centers is necessary for connection to back up facilities (e.g. for database synchronization), to other Control Centers (e.g., for coordination), or to other platforms inside the perimeter of the utility (e.g., outage management) or outside this perimeter (e.g. energy market platform). The primary purpose of the Inter-Control Center Communications is to transfer data between platforms for data synchronization, status reporting, and cross-platform data access.

These communications are generally assured through the Inter-Control Center Protocol (ICCP) standardized as IEC 60870-6 (Telecontrol Application Service Element TASE-2) protocol, although earlier protocols may still be in use in certain older systems.

ICCP uses an underlying transport-service, normally TCP/IP over Ethernet. The traffic is by nature sporadic with an exchange volume which can be very large for example for main to back up control center links. The delay requirement is often of the order of hundreds of milliseconds or more depending on the applications. Lower delays may be needed for much shorter control signals. The required throughput and the consequent capacity are determined by the maximum time required for completing a large data exchange (for example a main-back up synchronization). Dedicated resources such as Ethernet-over SDH with NxE1 capacity or an Ethernet pseudo-wire over MPLS are generally allocated to these higher traffic links, although much lower capacity links have been used in the past.

Many inter-platform applications operate beyond the reach of the power utility dedicated telecom infrastructure. The communication links are therefore provisioned through public telecom service providers. Security in this case, is a more critical issue than transfer time, considering that an inadequately protected ICCP connection forms an open door to the control of the energy network.

Remote connection of Operator positions to the Control Center platform also requires high-speed communications. An Ethernet connection with a throughput of 2-10 Mbps generally allows an adequate quality communication link for connecting these remote workstations. 